\title{
Graphene Oxide Nanosheets Reshape Synaptic Function in Cultured Brain Networks
}

Rossana Rauti $i^{\dagger}$,Neus Lozano ${ }^{\S}$, Veronica León ${ }^{\ddagger}$, Denis Scaini ${ }^{\dagger, \#}$, Mattia Musto ${ }^{\varpi}$, Ilaria Rago ${ }^{\#}$, Francesco P. Ulloa Severino ${ }^{\circ}$, Alessandra Fabbro" ${ }^{\text {, Loredana Casalis }}{ }^{\#}$, Ester Vázquez ${ }^{\dagger}$, Kostas Kostarelos $^{\S}$, Maurizio Prato ${ }^{\| \notin z^{*}}$ and Laura Ballerini ${ }^{\dagger, \sigma^{*}}$

\section{Supporting Results}

\section{Figure S1}

a

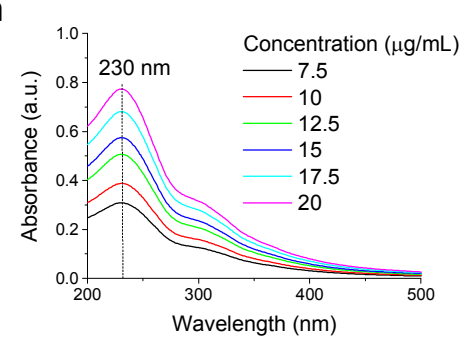

b

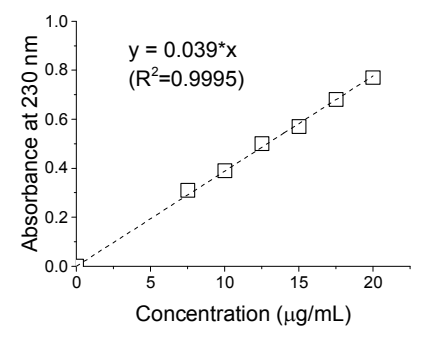

C

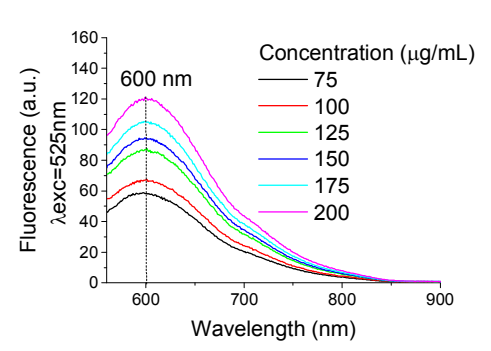

d

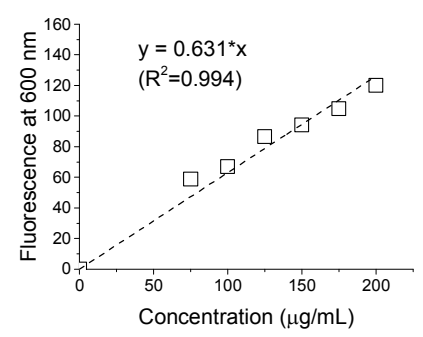

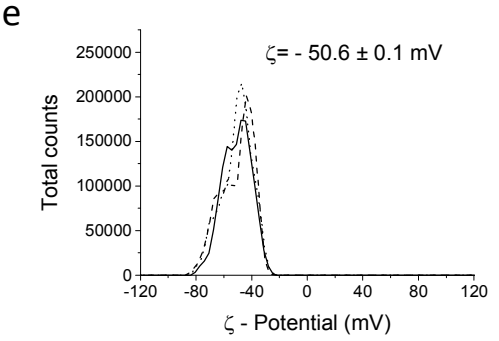

f

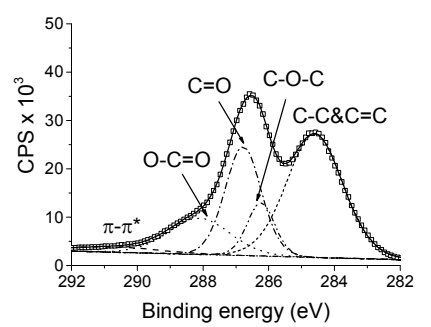

Figure S1. Physicochemical characterization of s-GO. (a) UV-Vis absorption spectra and (b) maximum absorbance at 230nm. (c) Fluorescence spectra and (d) maximum fluorescence at 600nm. (e) Zeta-potential measurements. (f) High resolution Cls XPS spectrum. 


\section{Figure S2}

a

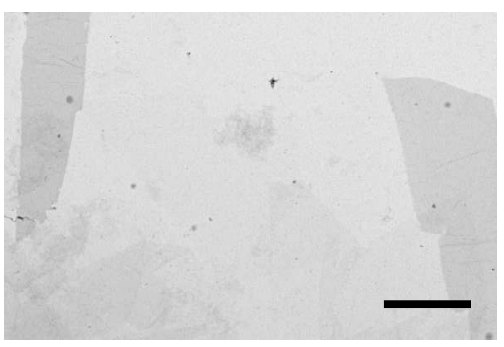

b

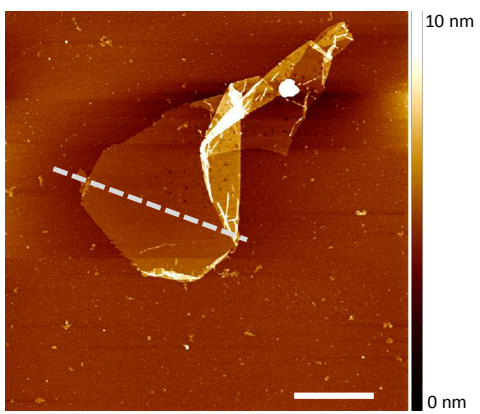

C

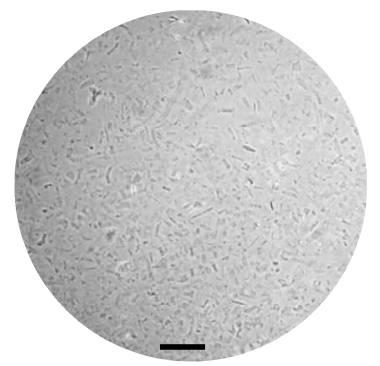

d

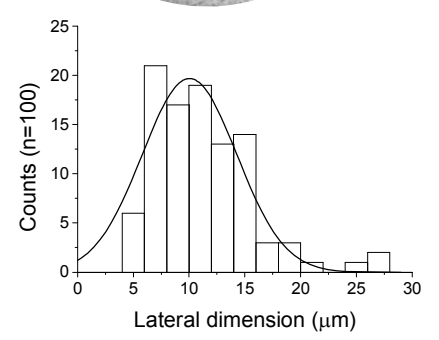

e

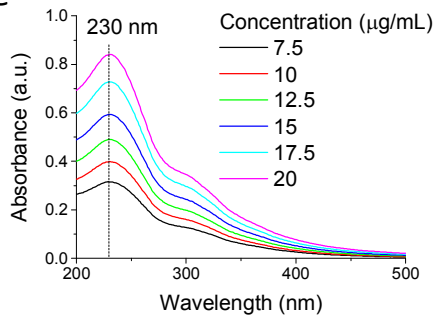

f

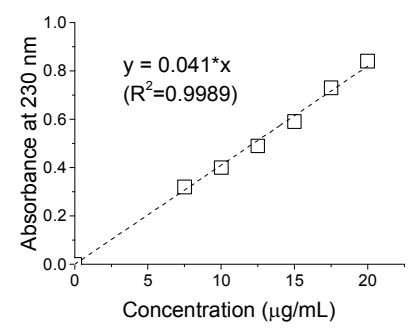

g

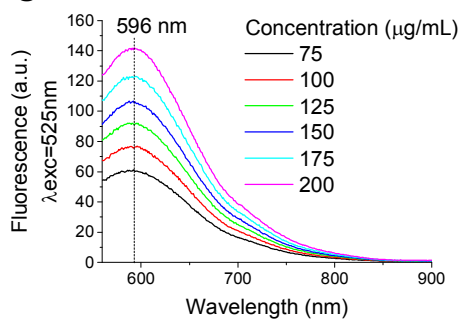

h

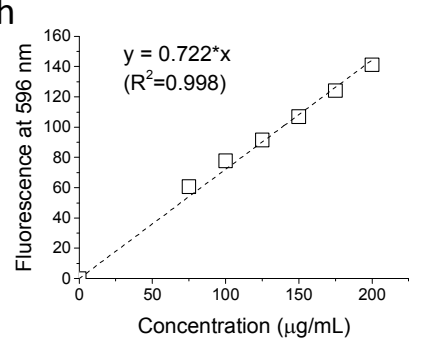

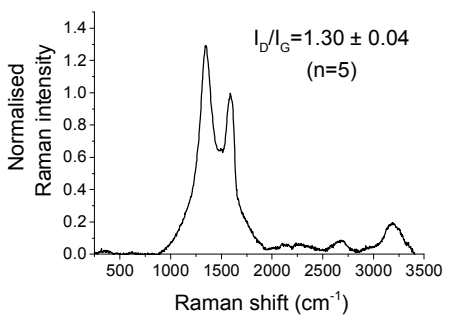

j

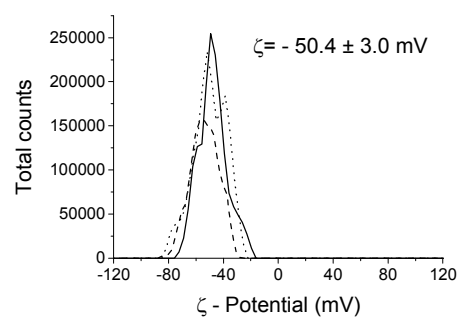

k
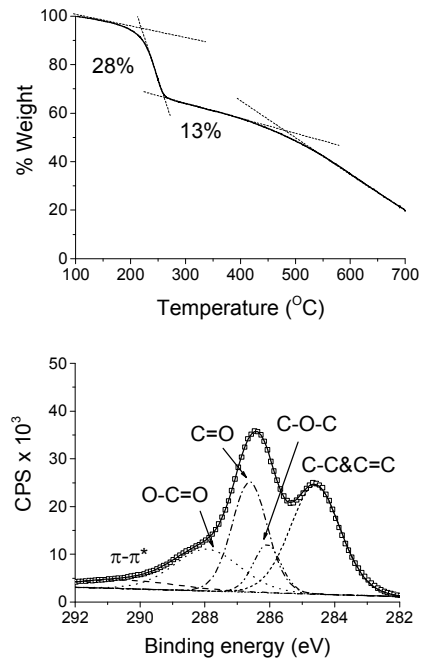

Figure S2. Physicochemical characterization of l-GO. (a) TEM micrograph (scale bar 5 $\mu$ ). (b) AFM height image (scale bar $2 \mu \mathrm{m}$. (c) Bright field optical microscopy image (scale bar $50 \mu \mathrm{m}$ ) and (d) lateral dimension distribution analysis. (e) UV-Vis absorption spectra and (f) maximum absorbance at 230nm (g) Fluorescence spectra and (h) maximum fluorescence at 596nm. (i) Normalized Raman spectrum. (j) Zetapotential measurements. (k) TGA analysis. (l) High resolution C1s XPS spectrum. 
Table S1. Summary of the physicochemical characterization of l-GO and s-GO

\begin{tabular}{|c|c|c|c|}
\hline & Technique & $l$-GO & $s$-GO \\
\hline \multirow[t]{3}{*}{ Lateral dimension (*) } & Optical microscopy & $5 \mu \mathrm{m}-30 \mu \mathrm{m}$ & - \\
\hline & TEM & $10-30 \mu \mathrm{m}$ & $200 \mathrm{~nm}-1 \mu \mathrm{m}$ \\
\hline & AFM & $10-15 \mu \mathrm{m}$ & $50 \mathrm{~nm}-500 \mathrm{~nm}$ \\
\hline Thickness & $A F M$ & $1 \mathrm{~nm}$ (1 layer) & $1.3 \pm 0.3 \mathrm{~nm}$ (1-2 layers) \\
\hline \multirow[t]{2}{*}{ Optical properties } & Absorbance & $A_{230}=0.041 * C_{G O}(\mu \mathrm{g} / \mathrm{mL})$ & $A_{230}=0.039 * C_{G O}(\mu \mathrm{g} / \mathrm{mL})$ \\
\hline & Fluorescence, $\lambda_{\text {exc }} 525 \mathrm{~nm}$ & $\mathrm{~F}_{596}=0.722 * \mathrm{C}_{\mathrm{GO}}(\mu \mathrm{g} / \mathrm{mL})$ & $\mathrm{F}_{600}=0.631 * \mathrm{C}_{\mathrm{GO}}(\mu \mathrm{g} / \mathrm{mL})$ \\
\hline Degree of defects $\left(I_{D} / I_{G}\right)$ & Raman & $1.30 \pm 0.04$ & $1.35 \pm 0.02$ \\
\hline Surface charge & $\zeta$-potential & $-50.4 \pm 3.0 \mathrm{mV}$ & $-50.6 \pm 0.1 \mathrm{mV}$ \\
\hline Functionalization degree & $T G A$ & $41 \%$ & $41 \%$ \\
\hline Chemical composition (Purity) & $X P S$ & C: $68.8 \%, 0: 30.8 \%, \mathrm{~N}: 0.4 \%(99.6 \%)$ & C: $69.9 \%, 0: 29.6 \%, \mathrm{~N}: 0.5 \%(99.5 \%)$ \\
\hline C:O ratio & $X P S$ & 2.2 & 2.3 \\
\hline$\pi-\pi^{*}, O-C=O, C=O, C-O-C, C=C$ & $X P S$ & $4.3 \%, 13.2 \%, 18.7 \%, 6.4 \%, 26.2 \%$ & $2.2 \%, 10.3 \%, 18.3 \%, 6.8 \%, 32.3 \%$ \\
\hline
\end{tabular}

(*) Lateral dimensions of $l-G O$ and $s$-GO are reported as a range between the minimum and the maximum size detected.

\section{Table S2. XPS analysis of graphite, l-GO and s-GO.}

(a) XPS survey spectra for elemental analysis in $O 1 s, N 1 s, C 1 s$ and $\mathrm{Si} 2 \mathrm{p}$ regions and (b) XPS high resolution $C 1$ s spectra for the quantification of the $\pi-\pi^{*}, O-C=O, C=O, C-O-C, C-O H$ and $C-C \& C=C$ contributions.

\begin{tabular}{|c|c|c|c|c|c|c|c|c|c|}
\hline System & $O 1 \mathrm{~s}$ & $N 1 s$ & $C 1 \mathrm{~s}$ & Si $2 p$ & Purity & $C: O$ & & & \\
\hline Graphite & $3.0 \%$ & - & $96.3 \%$ & $0.7 \%$ & $99.3 \%$ & 32.1 & & & \\
\hline$l-G O$ & $30.8 \%$ & $0.4 \%$ & $68.8 \%$ & - & $99.6 \%$ & 2.2 & & & \\
\hline$s-G O$ & $29.6 \%$ & $0.5 \%$ & $69.9 \%$ & - & $99.5 \%$ & 2.3 & & & \\
\hline System & \multicolumn{2}{|c|}{$\pi-\pi^{*}$} & \multicolumn{2}{|c|}{$O-C=O$} & \multicolumn{2}{|c|}{$C=O$} & $C-O-C$ & $\mathrm{C}-\mathrm{OH}$ & $C-C \& C=C$ \\
\hline Graphite & \multicolumn{2}{|c|}{$289.9 \mathrm{eV} 15.1 \%$} & \multicolumn{2}{|c|}{-} & \multicolumn{2}{|c|}{-} & - & $285.6 \mathrm{eV} 18.5 \%$ & $284.6 \mathrm{eV} 62.7 \%$ \\
\hline$l-G O$ & \multicolumn{2}{|c|}{$290.5 \mathrm{eV} \quad 4.3 \%$} & \multicolumn{2}{|c|}{$287.9 \mathrm{eV} 13.2 \%$} & \multicolumn{2}{|c|}{$286.6 \mathrm{eV} 18.7 \%$} & $286.1 \mathrm{eV} 6.4 \%$ & $-\quad-$ & $284.6 \mathrm{eV} 26.2 \%$ \\
\hline$s-G O$ & $290.7 \mathrm{eV}$ & $2.2 \%$ & $288.1 \mathrm{eV}$ & $10.3 \%$ & $286.8 \mathrm{eV}$ & $18.3 \%$ & $286.2 \mathrm{eV} 6.8 \%$ & - & $284.6 \mathrm{eV} 32.3 \%$ \\
\hline
\end{tabular}


Figure S3.
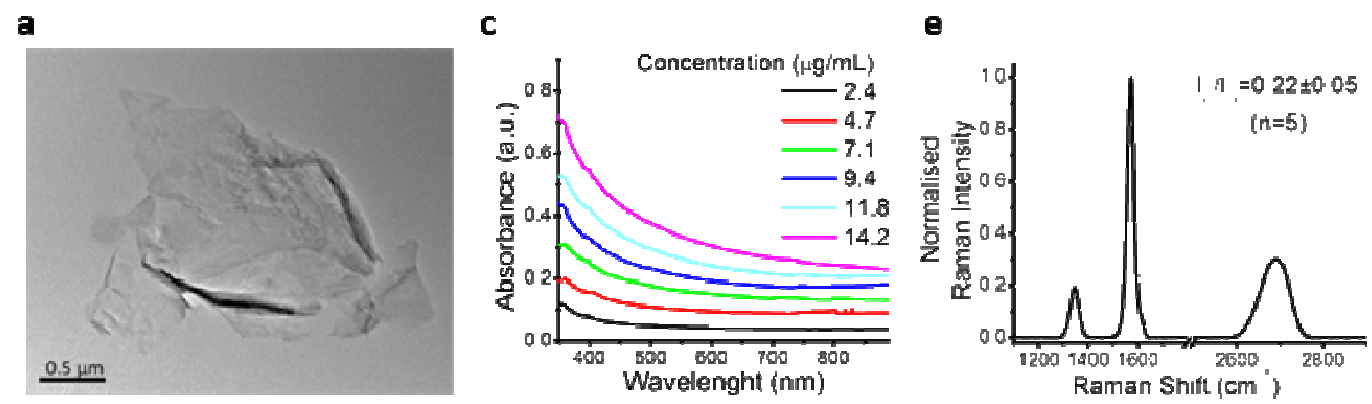

b

d
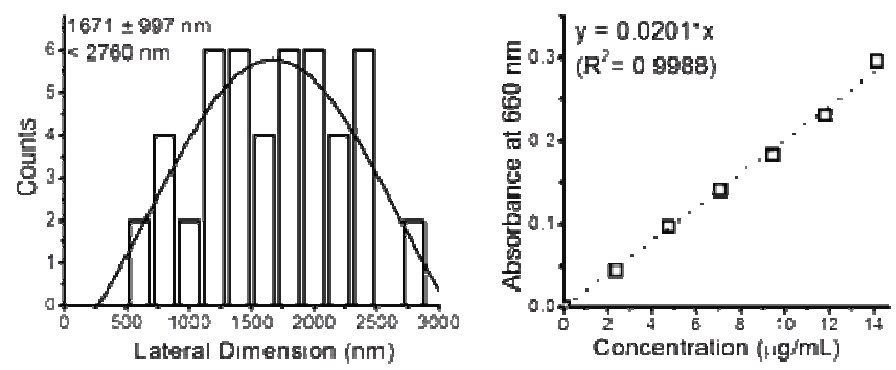

f

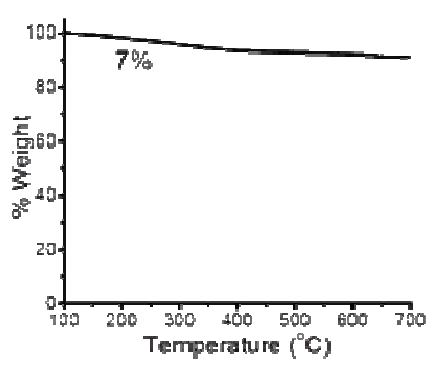

Figure S3. Physicochemical characterization of GR: (a) TEM micrograph and (b) lateral dimension distribution analysis. (c) UV-Vis absorption spectra and (d) absorbance at 660nm. (e) Normalized Raman spectrum at $532 \mathrm{~nm}$. (f) TGA analysis. 
Figure S4.

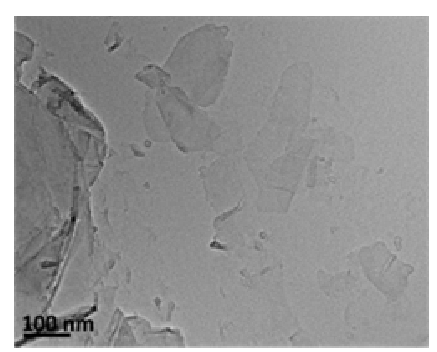

b

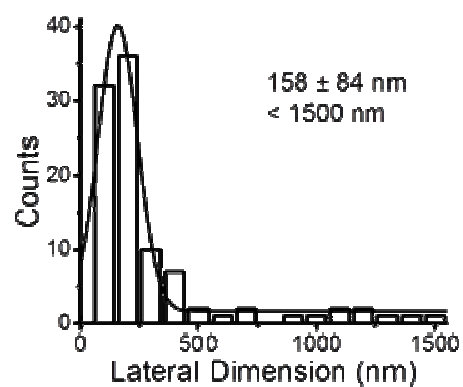

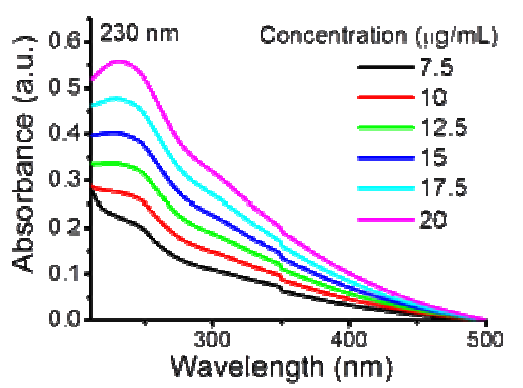

d

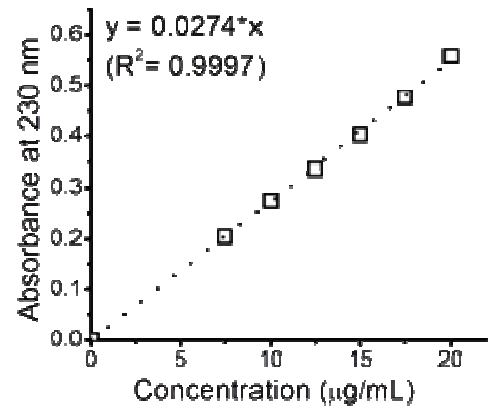

e

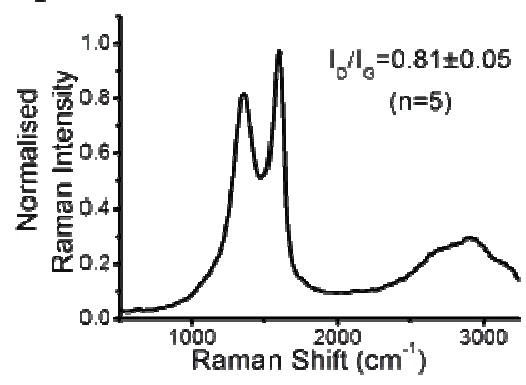

f

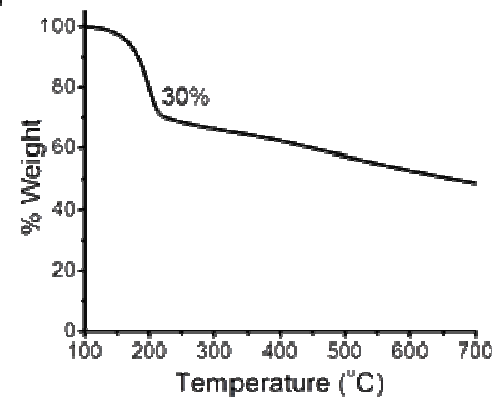

Figure S4. Physicochemical characterization of commercially-available GO (A-GO). (a) TEM micrograph and (b) lateral dimension distribution. (c) UV-Vis absorption spectra and (d) maximum absorbance at 230nm. (e) Normalized Raman spectrum at $532 \mathrm{~nm}$. (f) TGA analysis. 


\section{A-GO effects on synaptic transmission}

To validate our results on s-GO interference with synapses we tested the Antolin-GO (AGO) in a separated set of experiments where hippocampal cultures were treated for 6-8 days with A-GO $(1 \mu \mathrm{g} / \mathrm{mL}$ and $10 \mu \mathrm{g} / \mathrm{mL})$. Membrane capacitance and input resistance displayed similar values in all the tested treatments, summarized in Table S2.

\begin{tabular}{|c|c|c|}
\hline & Capacitance $(\mathrm{pF})$ & Input Resistance $(\mathrm{M} \Omega)$ \\
\hline Control $_{1} \quad \mathrm{n}=17$ & $47 \pm 5$ & $1078 \pm 168$ \\
\hline $\mathrm{A}^{-\mathrm{GO}_{1}} \mathrm{n}=22$ & $49 \pm 4$ & $997 \pm 158$ \\
\hline Control $_{10} \quad \mathrm{n}=11$ & $79 \pm 11$ & $679 \pm 80$ \\
\hline $\mathrm{A}-\mathrm{GO}_{10} \quad \mathrm{n}=22$ & $70 \pm 10$ & $1011 \pm 130$ \\
\hline
\end{tabular}

Figure S5 shows representative tracings of the recorded electrical activity. As for s-GO, also in this case, in neurons exposed to low $(1 \mu \mathrm{g} / \mathrm{mL})$ A-GO, the amplitude and frequency of PSCs were not affected compared to control cultures $(71 \pm 8 \mathrm{pA}$ and $2.5 \pm 0.3 \mathrm{~Hz}$ control, $\mathrm{n}=17$; $53 \pm 5 \mathrm{pA}$ and $2.7 \pm 0.4 \mathrm{~Hz}$ for A-GO, $\mathrm{n}=22$; summarized in Figure S5 plots). When incubating neurons with higher A-GO concentrations $(10 \mu \mathrm{g} / \mathrm{mL})$, we detected a significant decrease $(\mathrm{P}<$ 0.001; Student's t-test) in PSCs frequency when compared to control cultures $(0.4 \pm 0.2 \mathrm{~Hz}, \mathrm{n}=22$ in A-GO and $2.8 \pm 0.5 \mathrm{~Hz}$ in control, $\mathrm{n}=11$ ). The PSCs amplitude was not significantly different between the two conditions (data are summarized in Figure S5, top plots).

Both neuronal and glial densities were not affected by $10 \mu \mathrm{g} / \mathrm{mL}$ A-GO ( $\mathrm{n}=7$ visual fields; see plots in Figure S5, bottom panel). Neuronal activity was then monitored by calcium imaging in $10 \mu \mathrm{g} / \mathrm{mL}$ A-GO condition. Like s-GO-treatment, in A-GO treated cultures the $50 \%(\mathrm{n}=2$ out of 4$)$ of the recorded fields didn't show any activity. In our recordings, spontaneous $\mathrm{Ca}^{2+}$ signaling is detected in $40 \%$ ( 6 out of 14 cells, control, $n=2$ fields) and similarly, in 38\% (6 out of 15 cells, A$\mathrm{GO}, \mathrm{n}=2$ fields) of the cells visualized in each field. Control $\mathrm{Ca}^{2+}$ oscillations displayed an IEI of $50 \pm 5 \mathrm{~s}$ ( $\mathrm{n}=6$ cells $)$ that was significantly lower $(\mathrm{P}<0.001$; Student's t-test $)$ than that measured in A-GO treated cultures $(168 \pm 11 \mathrm{~s}, \mathrm{n}=6$ cells $)$. When we measured IEI in the presence of bicuculline $(20 \mu \mathrm{M}, 20 \mathrm{~min})$, the control value was still significantly lower $(\mathrm{P}<0.001$; Student's ttest) than that measured in A-GO treated cultures ( $26 \pm 3 \mathrm{~s}, \mathrm{n}=6$ control cells, vs $108 \pm 10 \mathrm{~s}, \mathrm{n}=6$ GO cells). 


\section{Figure S5}

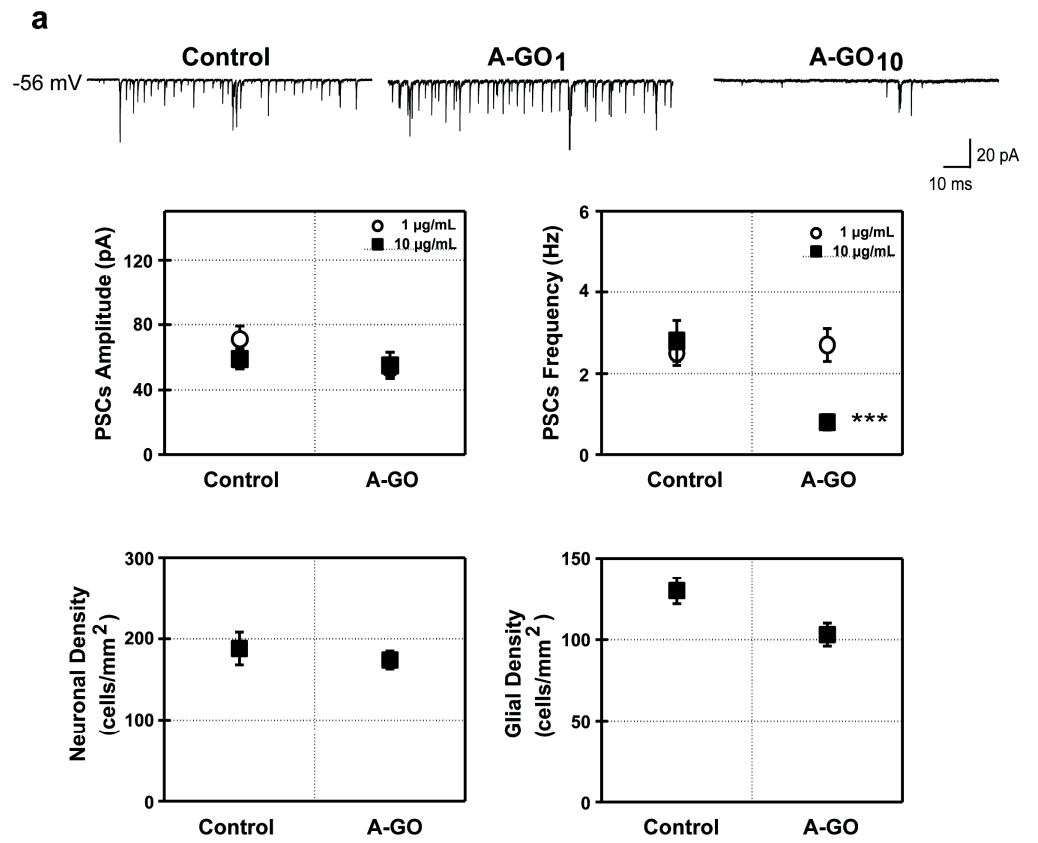

Figure S5. Spontaneous synaptic activity recorded from hippocampal neurons in control and A-GO at 1 $\mu \mathrm{g} / \mathrm{mL}$ and $10 \mu \mathrm{g} / \mathrm{mL}$ grown for $8 \div 10$ DIV. PSCs were detected at $-56 \mathrm{mV}$ holding potential. Top plots represent pooled data and summarize average PSCs amplitude and frequency: note the reduction in A-GOtreatment $(10 \mu \mathrm{g} / \mathrm{mL}$, final concentration) of PSCs frequency $(* * *=P<0.001$ Student's test, data are mean $\pm S E M)$. Bottom plots summarize neuronal (left) and glial (right) densities in both conditions: no significant differences were detected.

\section{Inhibitory synaptic activity analysis}

To determine how s-GO $(10 \mu \mathrm{g} / \mathrm{mL})$ treatment influenced GABAergic transmission we used simultaneous whole-cell recordings from pairs of closely located interconnected neurons (Figure $\mathrm{S}^{1}$ ). The GABAergic nature of the evoked events was confirmed by the measured PSC slow decay $\left(\tau=24 \pm 0.9 \mathrm{~ms}, \mathrm{n}=16^{1}\right)$ and sensitivity to bicuculline $(20 \mu \mathrm{M})$ application $(\mathrm{n}=8)$. Apparently s-GO did not affect the density of inhibitory synapses, in fact the probability of finding monosynaptic coupled pairs was 70\% (10 out of 14 pairs) in control and $66 \%$ in GO (6 out of 9 pairs). The similar GABAergic innervation was associated with similar amplitude of $\mathrm{GABA}_{\mathrm{A}}$ mediated events (on average the peak amplitude of evoked PSCs was $89 \pm 16$ pA and $96 \pm 22 \mathrm{pA}$ in control and s-GO treated neurons, respectively). We indirectly assessed the GABA release probability $\left(p_{r}\right)$ in controls and s-GO treated pairs by measuring the paired-pulse $(20 \mathrm{~Hz})$ ratio [PPR, calculating the ratio between the mean peak amplitude of the second and the first $\left.\mathrm{PSC}^{2,3}\right]$. In 
both culture groups, pairs showed similar short-term depression (PPR $0.7 \pm 0.12$ and $0.5 \pm 0.18$, control and s-GO treated pairs, summarized in Figure S6a) thus ruling out gross differences in $p_{r}$.

\section{Inhibitory synaptic density analysis}

To further rule out s-GO (10 $\mu \mathrm{g} / \mathrm{mL})$ affecting inhibitory synapses, we examined and compared GABAergic synapses densities between control and s-GO treated cultures (8-10 DIV) by co-immunostaining for the vesicular GABA transporter (VGAT) and for neuronal cytoskeletal component $\beta$-tubulin III (Figure S6b). The following primary antibodies were used: rabbit polyclonal anti- $\beta$ - tubulin III (Sigma T2200, 1:250 dilution) and mouse monoclonal antibody against the vesicular GABA transporter (VGAT, Synaptic Systems, 1:200 dilution). After the primary incubation, cultures were incubated with the secondary antibodies: Alexa 594 goat anti rabbit (Invitrogen, dilution 1:500) and Alexa 488 goat anti-mouse (Invitrogen, dilution 1:500). Confocal acquisition was performed at higher magnification (63x) and Z-stacks were acquired every $300 \mathrm{~nm}$ for both culture groups ( $\mathrm{n}=18$ fields for control and $\mathrm{s}-\mathrm{GO})$. As for the measurements related to VGLUT1-staining, offline analysis was performed using Volocity software and for each set of experiments the cell images were acquired using identical exposure settings. As shown in the plot in Figure S6b, no significant differences in VGAT immunoreactivity were found: control and s-GO-treated neurons displayed a similar number of VGAT positive puncta $(4.5 \times 10-4 \pm 2.6 \times 10-5$, control cultures; $4.0 \times 10-4 \pm 2.1 \times 10-5$, s-GO-treated cultures $)$. 


\section{Figure S6}

\section{a}
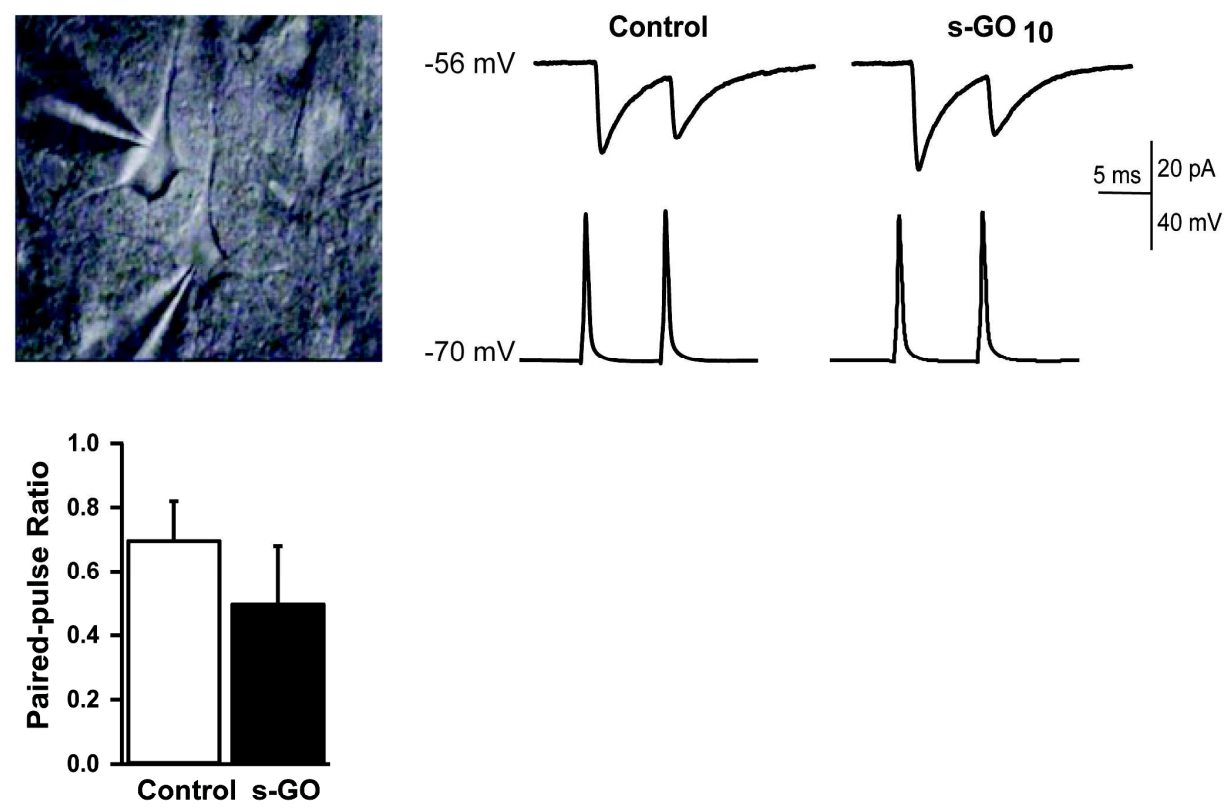

b
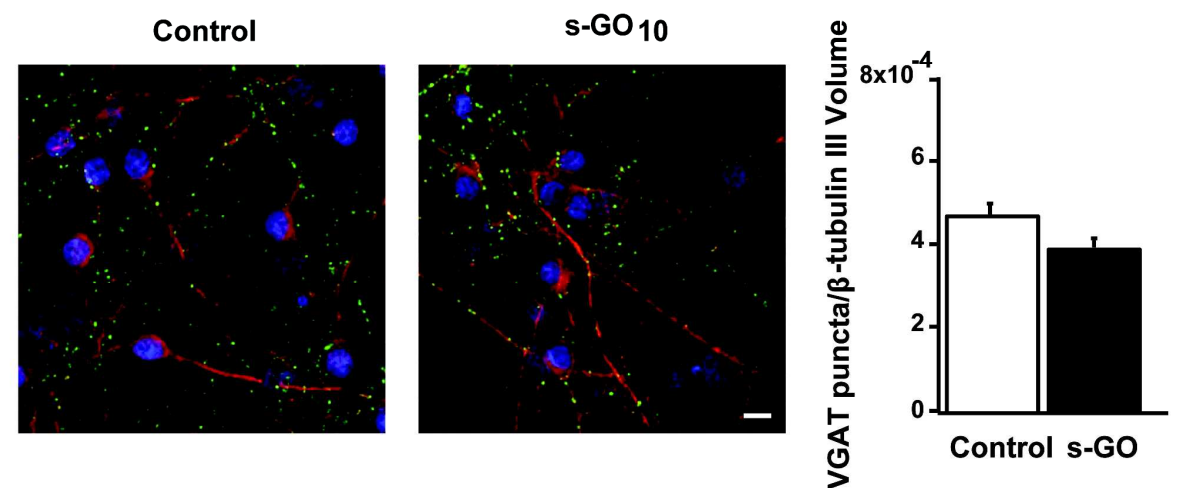

Figure S6 Representative bright field image depicts a pair of recorded neurons. Left tracings represent presynaptic pairs of action potentials (bottom) and the corresponding monosynaptic GABAergic evoked currents (top) in control and in s-GO-treated cultures. In the plot, histograms summarize the PPR values recorded in control and $s$-GO-treated cultures. In (b) confocal reconstructions ( $n=30 \mathrm{Z}$-stacks) showing hippocampal neurons treated and untreated with graphene oxide (10 $\mu \mathrm{g} / \mathrm{mL}$ final concentration) immunostained for the vesicular GABA transporter (VGAT, green) and counterstained for cytoskeletal components $\beta$-tubulin III (red). Scale bar: $10 \mu \mathrm{m}$. The plot shows no significant difference in terms of VGAT positive puncta normalized to $\beta$-tubulin volume $\left(4.5 \times 10^{-4} \pm 2.6 \times 10^{-5}\right.$, control cultures; $3.8 \times 10^{-4} \pm 2.1 \times$ $10^{-5}, s$-GO-treated cultures). 


\section{Pristine Graphene and MVs release}

Once we assessed the effect of s-GO on the release of the shedding MVs from glial cells, we decided to verify if also the pristine graphene (GR) could modulate it. We isolated MVs and quantify them by the analysis of the bands using western blot technique. Figure S7 shows the western blot analysis that was carried out on the P3 pellets purified from the supernatant collected from glial cultures previously incubated with GR $(10 \mu \mathrm{g} / \mathrm{mL})$. We have not found a positive signal from P3 pellet from glial cells incubated with GR and, as expected, no bands were obtained from P3 pellet purified from glial cultures not incubated (blot in Figure S7). We quantified astrocytes (GFAP positive cells; Figure S7 immunofluorescence images) density to determine cell survival (see Methods the Immunofluorescence staining paragraph for further details). We detected similar GFAP-positive cell densities in both GR treated and control groups (cell density GR: $855 \pm 23$ cells $/ \mathrm{mm}^{2}$; cell density control: $819 \pm 23$ cells $/ \mathrm{mm}^{2}$; summarized in the plot of Figure $\mathrm{S} 7$ ).

\section{Figure $\mathbf{3 7}$}
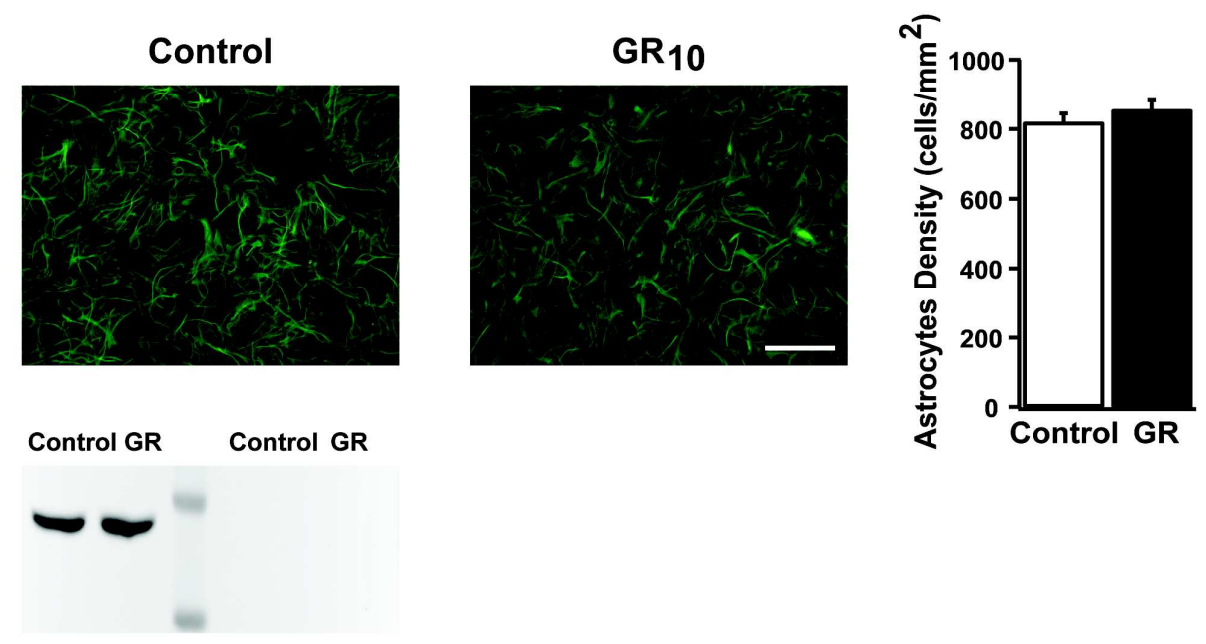

Figure S7. Top panel: GFAP (in green) immunolabeling of primary rat astrocytes cultures (3 weeks) incubated for 6 days with graphene pristine $(G R 10 \mu \mathrm{g} / \mathrm{mL})$ and control untreated cultures. Scale bar: 100 $\mu m$. The histograms show astrocyte cell density in incubated and control cultures. No statistical significance was found between the two conditions. Bottom panel: Western blotting of P3 pellets (right side) and cell lysates (left side) for MVs marker flotillin-1. Pellets were obtained from the medium of glial cultures incubated with GR or not. 


\section{s-GO flakes effects on synaptic activity in acute, 6-hours and 72-hours treatments}

To elucidate the timeline of s-GO effects on synaptic activity, we patch-clamped neurons and we positioned a second pipette identical to that used for patch-clamp recording at a distance of $200 \mu \mathrm{m}$ (under microscopy visual control) from the recorded cell. The second pipette was filled with standard saline solution (control, Krebs) or with s-GO (100 $\mu \mathrm{g} / \mathrm{mL}$ in Krebs solution). An injection of pressurized air (500 ms duration, 8 PSI; by a Picospritzer PDES-02DX; NPI electronic $\mathrm{GmbH}$, Germany) was used to deliver the s-GO acutely. The experimental settings were empirically tested. ${ }^{4}$ In our experiments we have estimated that a long pulse of pressure as the one used combined with the relatively large pipette distance resulted in a local (i.e. on the recorded neuron) concentration of s-GO of at least $10 \%$ of that contained in the pipette. Baseline PSCs were recorded before $(10 \mathrm{~min})$ and after $(10 \mathrm{~min})$ the local injection, as shown in Figure S8a: top, control tracings, where standard saline (Krebs) was ejected and bottom, s-GO ones, where s-GO in saline solution was pressure ejected. In neurons exposed to Krebs applications, spontaneous PSCs frequency did not change $(1.2 \pm 0.35 \mathrm{~Hz}$ before the pipette saline-ejection and $1 \pm 0.34 \mathrm{~Hz}$ after the pipette saline-ejection, $\mathrm{n}=17$; plot in Figure $\mathrm{S} 8 \mathrm{~b}$ ). On the contrary, acute s-GO ejection significantly increased $(* * * \mathrm{P}<0.001$ Student's test) the PSCs frequency (from a baseline of $0.8 \pm$ $0.35 \mathrm{~Hz}$ to a post injection frequency of $2.5 \pm 0.6 \mathrm{~Hz}, \mathrm{n}=21$, plot in Figure S8b). The increase in PSCs clearly emerged with a $10 \mathrm{~s}$ delay after the local s-GO ejection and was reversible, in fact PSCs frequency values returned to baseline values 7-8 min following the acute application. In all treatments, the PSCs amplitude was not affected. To note, GABAergic innervation recorded in pairs of closely located interconnected neurons was not affected by pressure ejection of s-GO applied under similar conditions ( $\mathrm{n}=10$; not shown).

The histograms in Figure S8c compare the frequency of PSCs when exposed to acute (pressure ejected) or to chronic s-GO $(10 \mu \mathrm{g} / \mathrm{mL})$. In the chronic experiments s-GO was added to the culturing medium and neuronal activity was recorded after 6-hours or after 72-hours. As shown in Figure S8c, after 6-hours we detected a slight increase in PSCs frequency when comparing control neurons $(1.15 \pm 0.4 \mathrm{~Hz}, \mathrm{n}=12)$ with $\mathrm{s}-\mathrm{GO}$ treated ones $(1.8 \pm 0.5 \mathrm{~Hz}, \mathrm{n}=15)$. However, already after 72 hours treatment s-GO induced a significant decrease (**P $<0.01$ Student's test) in PSCs frequency $(0.7 \pm 0.2 \mathrm{~Hz})$ in treated neurons $(\mathrm{n}=17)$ when compared with untreated control cells $(1.5 \pm 0.36 \mathrm{~Hz}, \mathrm{n}=14)$.

These experiments confirm the ability of s-GO to specifically target synapses. It is tempting to speculate that, in the long term, this acute interference is translated in the documented decrease in synaptic activity (after 3 days) and reduction in synaptic vesicles and synaptic contacts (after 6 days). 
a

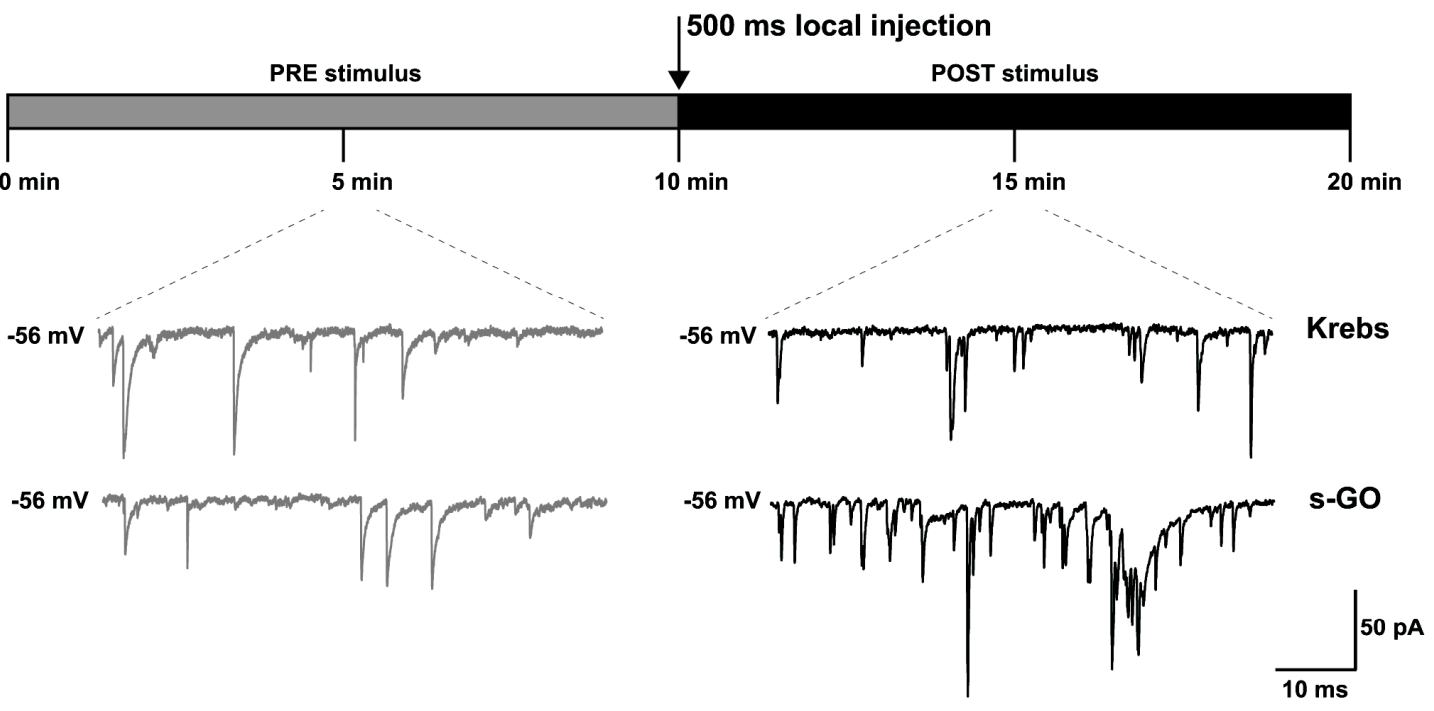

b

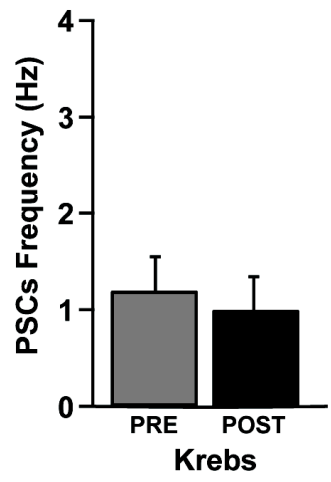

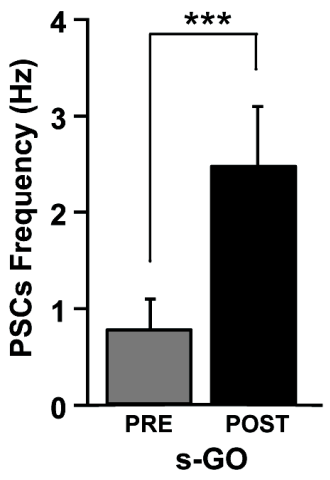

C

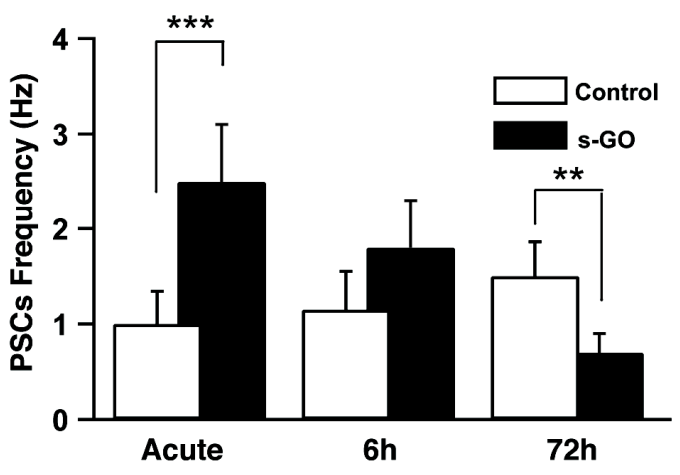

Figure S8. In (a) schematic experimental protocol and representative tracings of spontaneous synaptic activity recorded 5 min before and 5 min after control Krebs (top) or s-GO (bottom) pulses (500 ms). In (b) plots represent pooled data and summarize average PSCs frequency before and after Krebs (left) and s-GO (right) pulses $\left({ }^{* *} P<0.001\right.$ Student's test). In (c) histograms of pooled data represent the average PSCs frequency after acute injection (as in b) or after $6 \mathrm{~h}$ and $72 \mathrm{~h}$ chronic exposure to $\mathrm{s}-\mathrm{GO}(10 \mu \mathrm{g} / \mathrm{mL}$ final concentration) $(* * P<0.01$ Student's test).

\section{REFERENCES}

1. Cellot, G.; Toma, F.M.; Varley, Z.K.; Laishram, J.; Villari, A.; Quintana, M.; Cipollone, S.; Prato, M.; Ballerini, L. Carbon Nanotube Scaffolds Tune Synaptic Strenght in Cultured Neural Circuits: Novel Frontiers in Nanomaterial-Tissue Interactions. J Neurosci. 2011, 31, 12945-12953.

2. Manabe, T.; Wyllie, D.J.; Perkel, D.J.; Nicoll, R.A. Modulation of Synaptic Transmission and LongTerm Potentiation: Effects on Paired Pulse Facilitation and EPSC Variance in the CA1 Region of the Hippocampus. J Neurophysiol. 1993 70:1451-1459.

3. Debanne, D.; Guérineau, N.C.; Gähwiler, B.H.; Thompson, S.M. Paired-Pulse Facilitation and Depression at Unitary Synapses in Rat Hippocampus: Quantal Fluctuation Affects Subsequent Release. J Physiol. 1996 491,163-76.

4. Smith D.V.; Uteshev V.V. Heterogeneity of Nicotininc Acetylcholine Receptor Expression in the Caudal Nucleus of the Solitary Tract. Neuropharmacology 2008 54, 445-53. 